\title{
INVESTIGATION OF APPROPRIATE LADDER NUMBER ON PROBABILITY TABLE GENERATION
}

\author{
Kenichi Tada ${ }^{1}$ \\ ${ }^{1}$ Japan Atomic Energy Agency \\ Shirakata 2-4, Tokai-mura, Naka-gun, Ibaraki pref., 319-1195 Japan \\ tada.kenichi@jaea.go.jp
}

\begin{abstract}
The probability table method is widely used for continuous energy Monte Carlo calculation codes to treat the self-shielding effect in the unresolved resonance region. The ladder method is used to calculate the probability table. This method generates pseudo resonance structures using random numbers based on the averaged resonance parameters. The probability table affects the ladder number, i.e., the number of pseudo resonance structures. Since the higher the ladder number the longer it takes to create a cross-section library, reducing the value of the ladder number is an effective way to reduce the time spent in the creation of a crosssection library. However, the exact value of the most appropriate ladder number to use has not been found. Currently, users have to use a nuclear data processing system to manually set the ladder number to a large enough value in order to obtain a sufficiently converged probability table. In this study, the probability tables for all nuclides prepared in JENDL-4.0 are used to find the optimal ladder number. The results show that the probability table differences are small enough when the ladder number is 100 . The impact of these differences on neutronics calculations is found to be small.
\end{abstract}

KEYWORDS: probability table, ladder method, FRENDY, nuclear data processing

\section{INTRODUCTION}

The self-shielding effect in the unresolved resonance region has a large impact on fast and intermediate spectrum reactors [1]. The evaluated nuclear data library only provides the average values of the resonance spacing and the resonance partial widths in the unresolved resonance region [2]. The neutronics calculation codes cannot treat the self-shielding effect in the unresolved resonance region using directly the data from the evaluated nuclear data library.

There are several ways to account for the self-shielding effect in the unresolved resonance region [3]. The probability table method is widely used for continuous energy Monte Carlo calculation codes to treat the self-shielding effect in the unresolved resonance region. The probability table method uses the probability distribution of the cross-section in a given energy grid point which corresponds to the resonance structure. Currently, the probability table method is also used to calculate Bondarenko-style self-shielded cross sections that are used for multi-group deterministic calculation codes to treat the self-shielding effect in the unresolved resonance region [4]. Therefore, the probability table method is an effective method to use to treat the self-shielding effect in the unresolved resonance region for all neutronics calculations. 
The ladder method is used to generate the probability table $[4,5]$. The ladder method generates pseudo resonance structures using random numbers based on the averaged resonance parameters and calculates Doppler broadened cross sections in each pseudo resonance structure. Decreasing the time involved in making a probability table is very important because the ladder method requires a large amount of time.

Though the ladder number, i.e., number of pseudo resonance structures, has a large impact on the time spent in the creation of a cross-section library, an optimal ladder number has not been found. The PURR module in NJOY [6] is widely used to generate probability tables, but it cannot compare probability tables generated using different ladder numbers without modification, and this makes it difficult for PURR users to determine the most appropriate ladder number to use.

The Japan Atomic Energy Agency developed a new nuclear data processing system called FRENDY (FRom Evaluated Nuclear Data librarY to any application) [7]. The authors designed FRENDY to be extensible and implemented a function within FRENDY to compare probability tables generated using different ladder numbers. In this study, the optimal ladder number is found using this function. Neutronics calculation codes use probability tables to treat the self-shielding effect in the unresolved resonance region. The impact of the differences in probability tables on neutronics calculations is also investigated in this study.

\section{FINDING THE OPTIMAL LADDER NUMBER}

\subsection{Comparing probability tables}

A probability table consists of the probability, $P_{\mathrm{n}}$, and average cross section $\overline{\sigma_{n}}$ of the $n_{\text {th }}$ probability bin. $P_{\mathrm{n}}$ is the probability that total cross section in the unresolved resonance region satisfies following condition:

$$
\sigma_{t o t, n-1}<\sigma_{\text {tot }}(E) \leq \sigma_{t o t, n},
$$

where $\sigma_{t o t}(E)$ is the total cross section for which the incident neutron energy is $E$, and $\sigma_{t o t, n}$ is the boundary of $n_{\text {th }}$ probability bin. FRENDY and NJOY calculate $\sigma_{\text {tot }}(E)$ at the randomly selected energy grid point. The energy grid number of points in each ladder is 10,000 . The average cross section $\overline{\sigma_{n}}$ is the average of $\sigma_{\text {tot }}(E)$ that satisfies Eq. (1).

If $\sigma_{\text {tot }}(E)$ is calculated in all energy regions, then $P_{\mathrm{n}}$ is the probability that $\sigma_{\text {tot }}(E)$ satisfies Eq. (1) over the incident neutron energy range. When the peak resonance cross section is high, however, the energy range for which the incident neutron energy corresponds to the peak resonance cross section is very narrow. Analogously for the resonance cross section, $P_{\mathrm{n}}$ is small when $\sigma_{t o t, n}$ is high. Fig. 1 shows an example of a probability table and the average cross sections. It can be seen that the probability becomes small when the average cross section is large. Therefore, the probability table represented in Fig. 1 appropriately considers the resonance structure in the unresolved resonance region.

Furthermore, the figure shows that the probability at the top and bottom of the resonance is low. FRENDY and NJOY set the boundary of each probability bin so that each region within a bin is equally probable. The probability is low when the incident neutron energy is equal to the top or bottom of the resonance. However, such extremely high and low cross sections are important when considering the selfshielding effect. To appropriately treat the self-shielding effect, the boundary of a probability bin at the top and bottom of a resonance is different from the other regions so that each region within a bin is equally probable. 


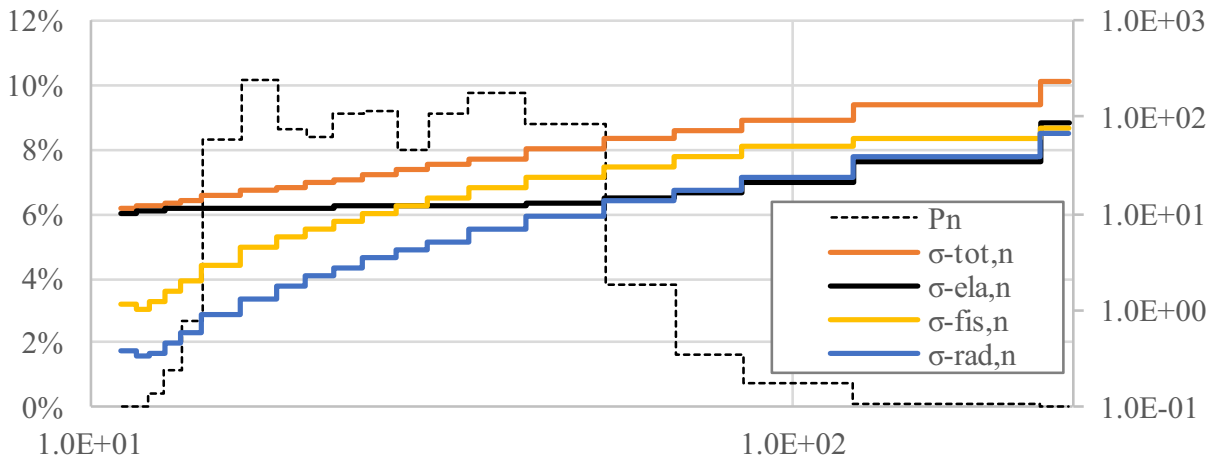

Figure 1. Probability table and average cross section of ${ }^{235} \mathrm{U}$ from JENDL-4.0. (Number of probability bin: 20, incident energy: $500 \mathrm{eV}$, temperature: 293.6 K)

The product of the probability table and the average cross section, $P_{r, n}$, is used to compare probability tables.

$$
P_{r, n}=P_{n} \times \frac{\overline{\sigma_{r, n}}}{\overline{\sigma_{r}}}
$$

where $r$ is a reaction type, and $\overline{\sigma_{r}}$ is the average cross section over all probability bins. $P_{r, n}$ is important in neutronics calculations. When a large cross-section difference is observed in the low probability region, this difference may not have a large impact on neutronics calculations. When the cross-section difference is small, and the probability is high, this difference may have an impact on neutronics calculations. $P_{r, n}$ is used in this work to study the differences in probability and average cross section.

The evaluated nuclear data library contains the energy-dependent average resonance parameters. For example, ${ }^{235} \mathrm{U}$ from JENDL-4.0 has 30 energy-dependent average resonance parameters. If the number of probability bins is 20 , there are 600 probability tables for ${ }^{235} \mathrm{U}$ from JENDL-4.0. It is difficult to compare all the probability table data. Therefore, the root mean square (RMS) value of the difference in all energy grids and all probability bins is used to find the optimal ladder number.

\subsection{Finding the optimal ladder number for a reference case}

The time involved in creating a probability table is proportional to the ladder number. It is difficult to use a huge number of ladder numbers as a reference case. I use ${ }^{235} U$ and ${ }^{238} U$ as a reference case for finding the optimal ladder number since these nuclides are the major nuclides for fast and intermediate spectrum reactors. The number of probability bins is 20 , and the reference ladder number is 50,000 . Tables I and II show the RMS values of the probability table difference for each ladder number in the reference case. As shown in Tables I and II, the RMS values are inversely proportional to the square root of ladder number, and may be taken to be the statistical error of the probability table.

The RMS values of the probability table become slightly larger as the temperature is increased. A comparison of the probability tables in each energy grid and each probability bin shows that the differences in $P_{n}$ become larger as the temperature is increased. The ladder method uses the identical resonance energy and resonance width even if the temperature is changed. The top and bottom of the resonance are smooth, and the boundary of the probability bin becomes narrow according to the resonance full width at half maximum (FWHM). Therefore, the variation of the probability table becomes larger when the temperature is increased. 
Generally, the tolerance value for linearization is $0.1 \%$. As shown in Tables I and II, the RMS values are less than $0.1 \%$ when the ladder number is larger than 300 . So, the RMS values are small enough when the ladder number is larger than 300 . Based on a reasonable computing time, it was decided that the ladder number for the reference case would be 1,000 .

Table I. RMS values of probability table difference in each ladder number $\left({ }^{235} \mathrm{U}\right)$.

\begin{tabular}{|r|c|c|c|c|c|c|c|c|c|c|c|c|}
\hline \multirow{2}{*}{$\begin{array}{c}\text { Ladder } \\
\text { No. }\end{array}$} & tot & ela & fis & rad & tot & ela & fis & rad & tot & ela & fis & rad \\
\hline 50 & $0.13 \%$ & $0.13 \%$ & $0.15 \%$ & $0.15 \%$ & $0.17 \%$ & $0.17 \%$ & $0.19 \%$ & $0.18 \%$ & $0.23 \%$ & $0.23 \%$ & $0.23 \%$ & $0.24 \%$ \\
\hline 100 & $0.10 \%$ & $0.10 \%$ & $0.10 \%$ & $0.11 \%$ & $0.13 \%$ & $0.13 \%$ & $0.13 \%$ & $0.13 \%$ & $0.15 \%$ & $0.15 \%$ & $0.15 \%$ & $0.15 \%$ \\
\hline 200 & $0.07 \%$ & $0.07 \%$ & $0.07 \%$ & $0.08 \%$ & $0.09 \%$ & $0.09 \%$ & $0.10 \%$ & $0.09 \%$ & $0.11 \%$ & $0.11 \%$ & $0.11 \%$ & $0.11 \%$ \\
\hline 300 & $0.06 \%$ & $0.06 \%$ & $0.06 \%$ & $0.06 \%$ & $0.08 \%$ & $0.07 \%$ & $0.08 \%$ & $0.08 \%$ & $0.09 \%$ & $0.09 \%$ & $0.09 \%$ & $0.09 \%$ \\
\hline 500 & $0.04 \%$ & $0.04 \%$ & $0.05 \%$ & $0.05 \%$ & $0.06 \%$ & $0.06 \%$ & $0.06 \%$ & $0.06 \%$ & $0.07 \%$ & $0.07 \%$ & $0.07 \%$ & $0.07 \%$ \\
\hline 1,000 & $0.03 \%$ & $0.03 \%$ & $0.04 \%$ & $0.04 \%$ & $0.04 \%$ & $0.04 \%$ & $0.05 \%$ & $0.05 \%$ & $0.05 \%$ & $0.05 \%$ & $0.05 \%$ & $0.05 \%$ \\
\hline 2,000 & $0.02 \%$ & $0.02 \%$ & $0.03 \%$ & $0.03 \%$ & $0.03 \%$ & $0.03 \%$ & $0.03 \%$ & $0.03 \%$ & $0.04 \%$ & $0.04 \%$ & $0.04 \%$ & $0.04 \%$ \\
\hline 5,000 & $0.02 \%$ & $0.02 \%$ & $0.02 \%$ & $0.02 \%$ & $0.02 \%$ & $0.02 \%$ & $0.02 \%$ & $0.02 \%$ & $0.02 \%$ & $0.02 \%$ & $0.02 \%$ & $0.02 \%$ \\
\hline 20,000 & $0.01 \%$ & $0.01 \%$ & $0.01 \%$ & $0.01 \%$ & $0.01 \%$ & $0.01 \%$ & $0.01 \%$ & $0.01 \%$ & $0.01 \%$ & $0.01 \%$ & $0.01 \%$ & $0.01 \%$ \\
\hline
\end{tabular}

Table II. RMS value of probability table difference in each ladder number $\left({ }^{238} \mathbf{U}\right)$.

\begin{tabular}{|r|c|c|c|c|c|c|c|c|c|c|c|c|}
\hline \multirow{2}{*}{$\begin{array}{c}\text { Ladder } \\
\text { No. }\end{array}$} & tot & ela & fis & rad & tot & ela & fis & rad & tot & ela & fis & rad \\
\hline 50 & $0.10 \%$ & $0.10 \%$ & $0.10 \%$ & $0.11 \%$ & $0.13 \%$ & $0.13 \%$ & $0.12 \%$ & $0.13 \%$ & $0.15 \%$ & $0.15 \%$ & $0.15 \%$ & $0.15 \%$ \\
\hline 100 & $0.07 \%$ & $0.07 \%$ & $0.07 \%$ & $0.08 \%$ & $0.09 \%$ & $0.09 \%$ & $0.09 \%$ & $0.09 \%$ & $0.10 \%$ & $0.10 \%$ & $0.10 \%$ & $0.10 \%$ \\
\hline 200 & $0.06 \%$ & $0.06 \%$ & $0.05 \%$ & $0.06 \%$ & $0.07 \%$ & $0.07 \%$ & $0.07 \%$ & $0.07 \%$ & $0.08 \%$ & $0.08 \%$ & $0.08 \%$ & $0.08 \%$ \\
\hline 300 & $0.04 \%$ & $0.04 \%$ & $0.04 \%$ & $0.05 \%$ & $0.05 \%$ & $0.05 \%$ & $0.05 \%$ & $0.06 \%$ & $0.06 \%$ & $0.06 \%$ & $0.06 \%$ & $0.06 \%$ \\
\hline 500 & $0.03 \%$ & $0.03 \%$ & $0.03 \%$ & $0.03 \%$ & $0.04 \%$ & $0.04 \%$ & $0.04 \%$ & $0.04 \%$ & $0.04 \%$ & $0.04 \%$ & $0.04 \%$ & $0.05 \%$ \\
\hline 1,000 & $0.02 \%$ & $0.02 \%$ & $0.02 \%$ & $0.03 \%$ & $0.03 \%$ & $0.03 \%$ & $0.03 \%$ & $0.03 \%$ & $0.03 \%$ & $0.03 \%$ & $0.03 \%$ & $0.03 \%$ \\
\hline 2,000 & $0.02 \%$ & $0.02 \%$ & $0.01 \%$ & $0.02 \%$ & $0.02 \%$ & $0.02 \%$ & $0.02 \%$ & $0.02 \%$ & $0.02 \%$ & $0.02 \%$ & $0.02 \%$ & $0.02 \%$ \\
\hline 5,000 & $0.01 \%$ & $0.01 \%$ & $0.01 \%$ & $0.01 \%$ & $0.01 \%$ & $0.01 \%$ & $0.01 \%$ & $0.01 \%$ & $0.01 \%$ & $0.01 \%$ & $0.01 \%$ & $0.02 \%$ \\
\hline 20,000 & $0.01 \%$ & $0.01 \%$ & $0.01 \%$ & $0.01 \%$ & $0.01 \%$ & $0.01 \%$ & $0.01 \%$ & $0.01 \%$ & $0.01 \%$ & $0.01 \%$ & $0.01 \%$ & $0.01 \%$ \\
\hline
\end{tabular}

\subsection{Finding the optimal ladder number for all nuclides prepared in JENDL-4.0}

The probability tables for each nuclide prepared in JENDL-4.0 are compared in order to determine the optimal ladder number. The number of probability bins is 20 , the temperature is $293.6 \mathrm{~K}$, and the reference ladder number is 1,000 . Figs. $2-5$ show the RMS values of the probability table difference for each ladder number for the reference case. The $x$-axis in Figs. $2-5$ is the ZA number [2], i.e., $1000 \times$ proton number $(\mathrm{Z})+$ atomic mass number $(\mathrm{A})$.

As shown in Figs. 2-5, the larger RMS values are observed for medium-heavy nuclides. The nuclides for which the RMS value (ladder number: 100 ) is larger than $0.2 \%$ are as follows:

$$
{ }_{28}^{59} \mathrm{Ni},{ }_{32}^{70} \mathrm{Ge},{ }_{32}^{72} \mathrm{Ge},{ }_{32}^{73} \mathrm{Ge},{ }_{32}^{74} \mathrm{Ge},{ }_{32}^{76} \mathrm{Ge},{ }_{34}^{82} \mathrm{Se},{ }_{38}^{88} \mathrm{Sr},{ }_{38}^{90} \mathrm{Sr},{ }_{52}^{130} \mathrm{Te},{ }_{53}^{135} \mathrm{I},{ }_{56}^{140} \mathrm{Ba},{ }_{80}^{204} \mathrm{Hg}
$$

One of the causes of the large difference is a large average level spacing. The average level spacing affects the distance between each resonance. If the average level spacing is large, the number of resonances in each pseudo resonance structure differs greatly. In such cases, some pseudo resonance structures overlap each resonance, and others do not. If a resonance is overlapped, the cross section at the bottom of the resonance is changed. Therefore, $P_{n}$ differs greatly from a ladder result to another ladder 
result when the average level spacing is larger. Since the variation of $P_{n}$ affects the statistical error of the probability table, the statistical error of the probability table becomes larger.

I found that the probability in each probability bin is different in some nuclides for which the average level spacing is large. FRENDY sets the boundary of a probability bin using the first ladder result so that each region within a bin is equally probable. When the resonance structure of the first ladder result is different than that of another ladder result, the probability in each bin is different. This indicates that the first ladder result may not be appropriate for setting the boundary of the probability bin when the average level spacing is large. When the average level spacing is large, not only the first but also other ladder results are required in order to set the boundary of a probability bin.

The results indicate that the RMS values of most nuclides are less than $0.1 \%$ when the ladder number is 100. This indicates that the differences in the probability tables are small enough when the ladder number is 100 . The time required to create a probability table for a nuclide (number of ladders: 100, number of temperatures: 1) was usually less than 1 hour. This is an acceptable amount of time to spend in the creation of a cross-section library.

As shown in Figs. 2-5, the RMS values depend on the nuclide and of the partial cross section involved. This indicates that the statistical error of the probability table of each nuclide is different when an identical ladder number is used. When creating probability tables, it's a good idea to reduce the statistical error as much as possible. This is currently being investigated.

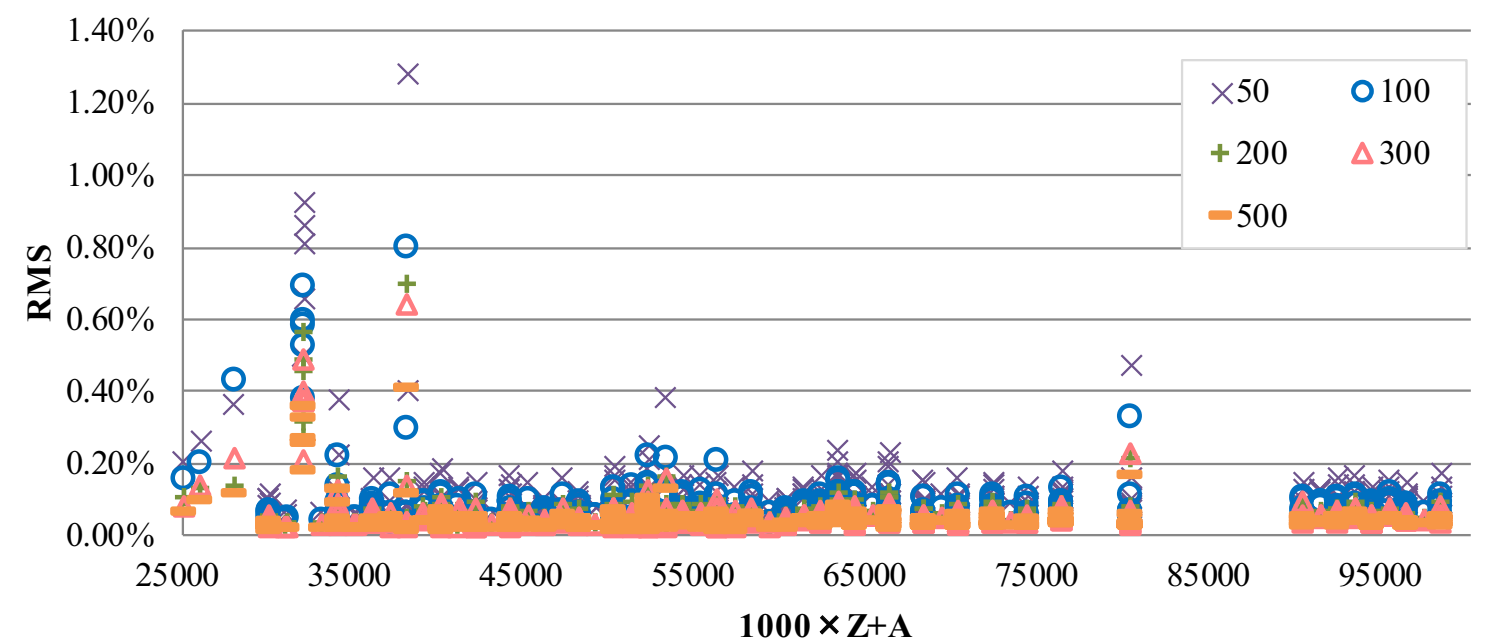

Figure 2. RMS values of probability table difference in each ladder number (total). 


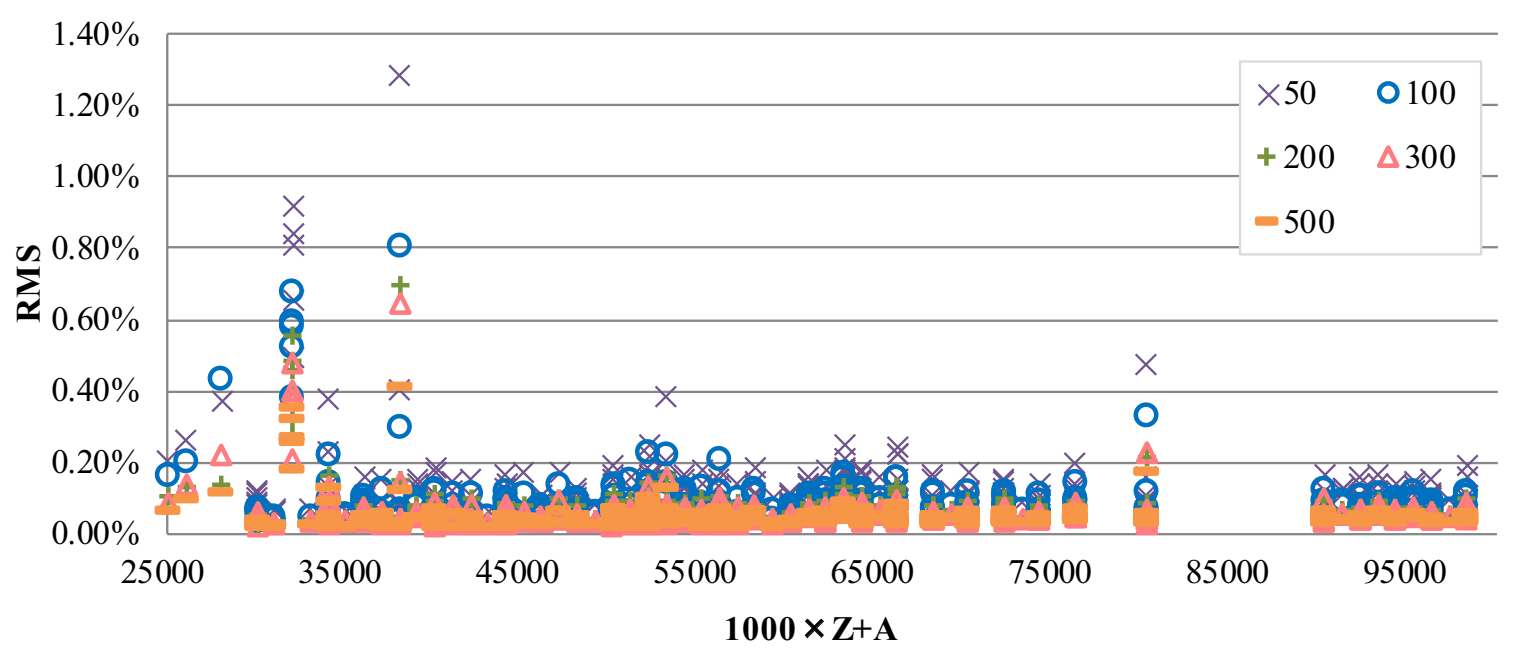

Figure 3. RMS values of probability table difference in each ladder number (elastic scattering).

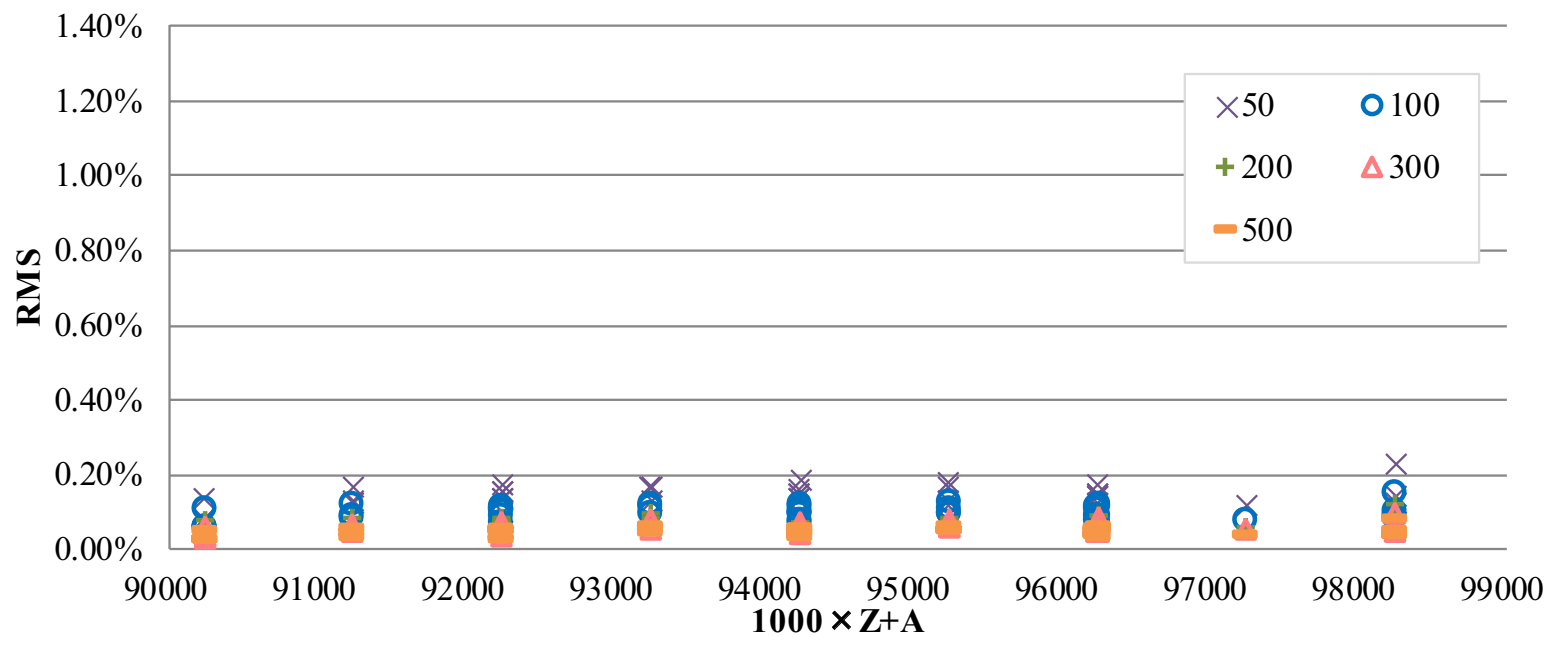

Figure 4. RMS values of probability table difference in each ladder number (fission).

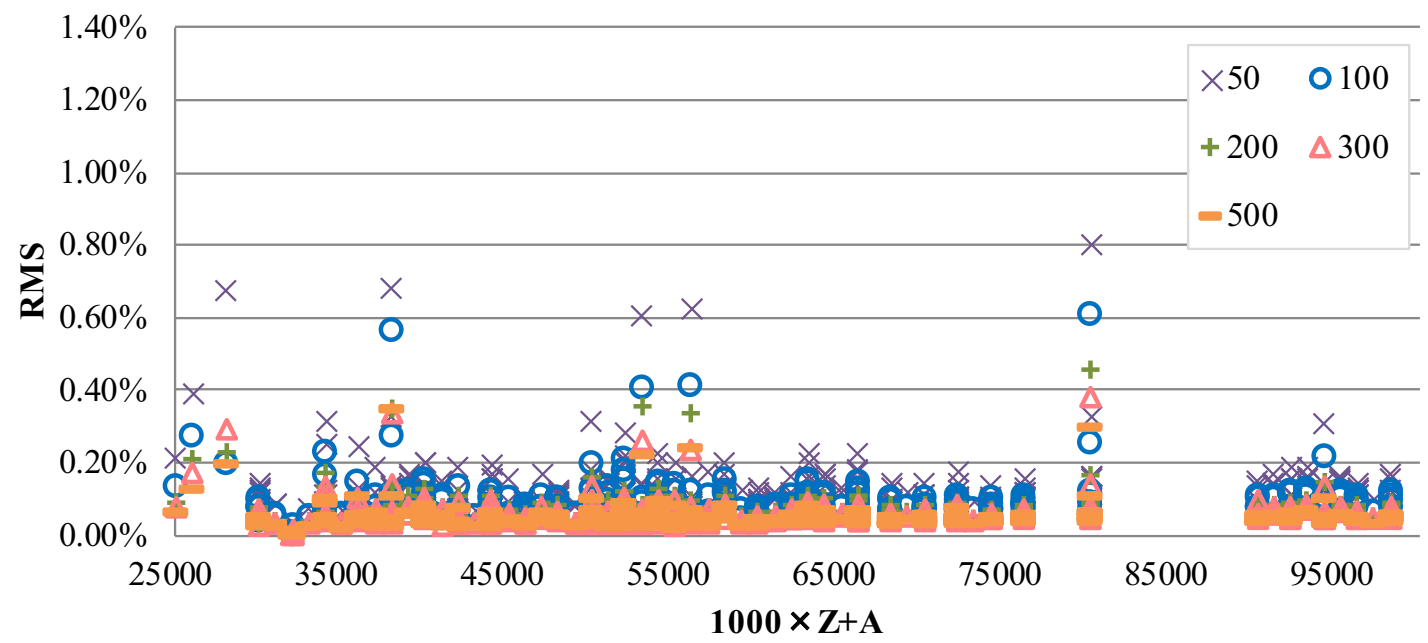

Figure 5. RMS values of probability table difference in each ladder number (radiation). 


\section{IMPACT ON NEUTRONICS CALCULATIONS}

To investigate the impact of probability table difference on neutronics calculations, I calculated the k-eff values for the benchmark experiments in the ICSBEP handbook [8]. A continuous energy Monte Carlo calculation code MCNP6 [9] was used for this calculation. The benchmark experiments that have a large sensitivity to the unresolved resonance region [10] were selected.

Fig. 6 shows the relative differences for the benchmark experiments. The ladder number of the reference calculation was 1,000. All processes used to generate the ACE file were carried out by FRENDY. The temperature, tolerance of the linearization, and number of probability bins were $293.6 \mathrm{~K}, 0.1 \%$, and 20 , respectively. The red line in Fig. 6 shows the statistical error $(1 \sigma)$.

As shown in Fig. 6, the relative difference is less than $1 \sigma$ in most cases. The relative difference is small enough even if the ladder number is 50 . This indicates that the probability table difference has only a small impact on neutronics calculations.

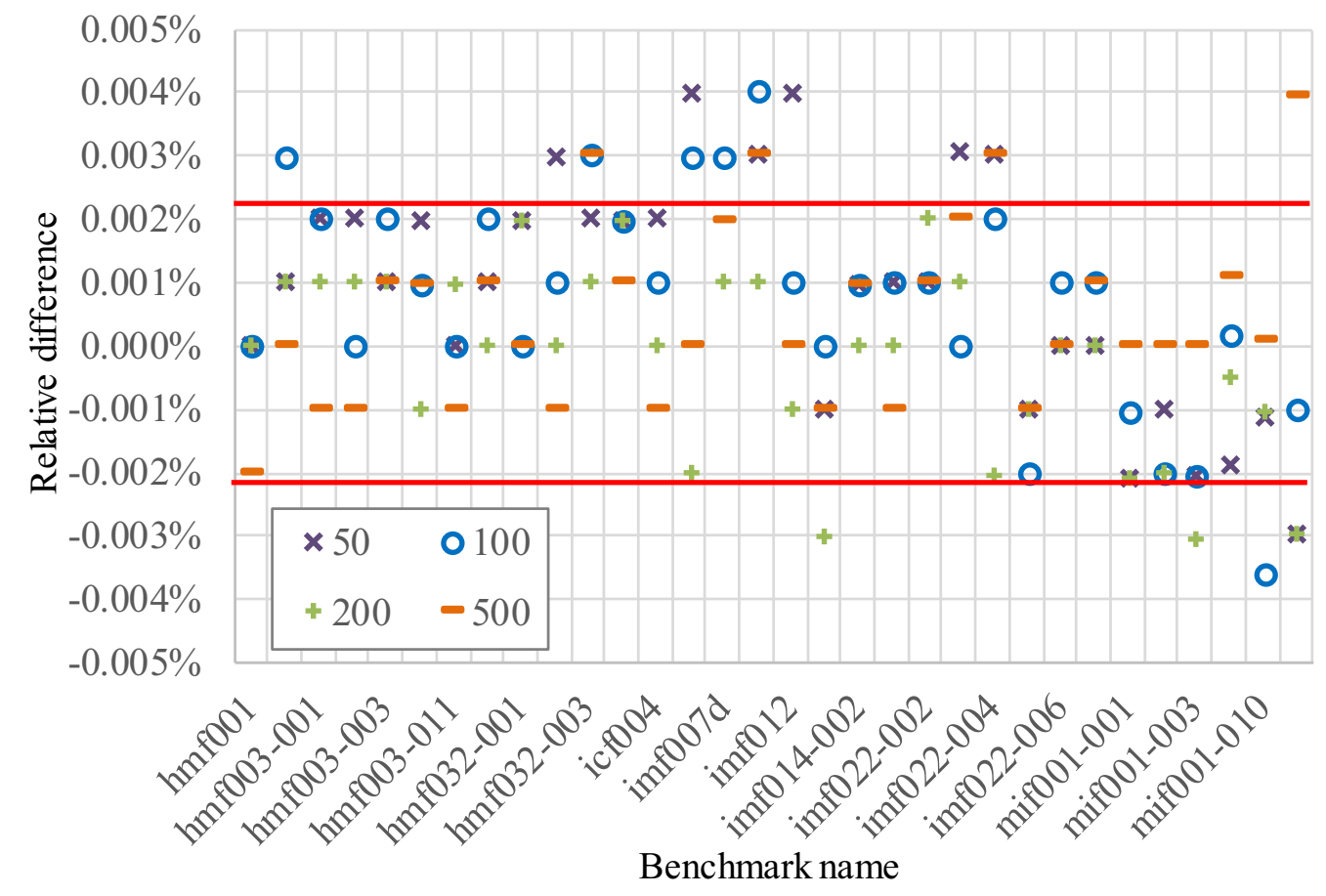

Figure 6. Comparison of k-eff values for ICSBEP benchmark in each ladder number.

\section{CONCLUSIONS AND FUTURE WORKS}

The probability table method is widely used for continuous energy Monte Carlo calculation codes to treat the self-shielding effect in the unresolved resonance region. In this study, the optimal ladder number is found in order to reduce the amount of time spent creating a cross-section library.

${ }^{235} \mathrm{U}$ and ${ }^{238} \mathrm{U}$ are used as a reference case for finding the optimal ladder number since these nuclides are the major nuclides for fast and intermediate spectrum reactors. The number of probability bins is 20 , and the reference ladder number is 50,000. The RMS values of probability table difference are small enough when the ladder number is larger than 300 . Based on the calculation time, it was decided that the ladder number for the reference case was 1,000 . 
The probability tables for all the nuclides prepared in JENDL-4.0 are used to find the optimal ladder number. The number of probability bins is 20 ; and the number of ladders is $50,100,200,300$, and 500 .

The results indicate that the probability table difference is small enough when the ladder number is 100 . Larger differences are observed for medium-heavy nuclides. One of the causes of the larger differences is a large average level spacing. The average level spacing has an impact on the pseudo resonance structure. Therefore, the statistical error of a probability table becomes large when the average level spacing is large.

To determine the impact of probability table difference on neutronics calculations, the criticality calculation results are compared. The integral experiments that have a large self-shielding effect are used for the comparison. The results indicate that probability table difference has only a small effect on neutronics calculations.

These results indicate that the optimal ladder number is 100 . This ladder number is used as the default value for generating probability tables in FRENDY.

An important value to consider when creating probability tables is the ladder number. However, the RMS values of probability table difference depend on the nuclide. When creating probability tables, it's a good idea to reduce the statistical error as much as possible. Calculation of the statistical error of the probability table is now under investigation.

\section{ACKNOWLEDGMENTS}

This work was supported by JSPS KAKENHI, Grant-in-Aid for Scientific Research (C) (18K05002).

\section{REFERENCES}

1. R.D. Mosteller and R.C. Little, "Impact of MCNP Unresolved Resonance Probability-Table Treatment on Uranium and Plutonium Benchmarks," Proc. ICNC'99, Versailles, France, Sep. 20-24, 1999, p. 341 (1999).

2. A. Trkov, M. Herman, D.A. Brown, “ENDF-6 Formats Manual,” BNL-203218-2018-INRE, Brookhaven National Laboratory (2018).

3. D.E. Cullen, "The importance of Resonance Self-Shielding," INDC(NDS)-0778, International Atomic Energy Agency (2019).

4. R.E. MacFarlane and A.C. Kahler, "Methods for Processing ENDF/B-VII with NJOY," Nucl. Data Sheets, 111, pp.2739-2890 (2010).

5. K. Tada, "Improvement of probability table generation using ladder method for a new nuclear data processing system FRENDY," Proc. Physor2018, Cancun, Mexico, Apr. 22-26, 2018 (2018).

6. A.C. Kahler (Ed.), “The NJOY Nuclear Data Processing System, Version 2016," LA-UR-17-20093, Los Alamos National Laboratory (2016).

7. K. Tada, S. Kunieda, Y. Nagaya, "Nuclear Data Processing Code FRENDY Version 1," JAEAData/Code 2018-014, Japan Atomic Energy Agency (2019).

8. OECD/NEA, "International Handbook of Evaluated Criticality Safety Benchmark Experiments," NEA/NSC/DOC(95)03 (2014).

9. D.B. Pelowitz (Ed.), "MCNP6 USER'S MANUAL Version 1.0," LA-CP-13-00634 Rev. 0, Los Alamos National Laboratory (2013).

10. A. Trkov, "Status of the ACE Verification Project," Technical Meeting on Nuclear Data Processing, IAEA https://www-nds.iaea.org/index-meeting-crp/TMDataProc/docs/Trkov_ACE_Verification_Project.pdf, (2018). 